\title{
A content analysis of the quality and behaviour change techniques of smartphone apps promoting the Mediterranean diet
}

\section{Abstract}

The popularity of smartphone apps is rising globally. However, the quality of widely available health apps and their effectiveness to promote behaviour change, by incorporating behavioural change techniques (BCTs) that have been suggested to result in behaviour change, is questionable. The aim of this study was to conduct a content analysis of smartphone apps that utilise the Mediterranean diet (MD), a dietary pattern linked to numerous health benefits, to promote healthy dietary behaviours. The specific objectives were to evaluate the quality of these apps and their use of BCTs. The iTunes Store and Google Play were searched for all available apps (free and requiring payment to download) promoting the MD. Apps were excluded if they were not available in English, did not focus only on the MD or the download process was corrupt. The Mobile App Rating Scale (MARS) was used to assess app engagement, functionality, aesthetics and information quality (the average of which represents a quality mean score (QMS)) and subjective quality. The Abraham \& Michie behaviour change taxonomy was adapted to create a 20-item checklist to assess the presence of BCTs in the eligible apps. Thirty nine apps (64.1\% of which were free to download) were analysed. The overall QMS was $2.96 \pm 0.46$. The lowest and highest scored QMS constructs were engagement (2.42 \pm 0.62$)$ and functionality $(3.95 \pm 0.45)$, respectively, whilst the overall mean subjective quality score was $1.71 \pm 0.51$ (scores' range 1-5). Apps were scored higher for aesthetics if they required payment (3.14 vs. 2.78, $\mathrm{p}=0.047$ ). Apps incorporated an average of $3.10 \pm 1.87 \mathrm{BCT}$, with no difference in the number of BCTs present according to platform or cost. From the five BCTs that have been suggested to promote behaviour change, 'self-monitoring' was present in six apps, 'intention formation' in 15 and 'goal setting' in one. None of the apps incorporated the 'review of behavioural goals' and 'feedback on performance' BCTs. The total QMS score was positively associated with the presence of BCTs $(r=0.409, p=0.008)$. Based on the MARS assessment, apps promoting the MD that are available in the public domain were of moderate quality. In contrast, the low presence, particularly of efficacious, BCTs and low subjective quality scores suggest that apps are actually of poor quality and potentially not effective in promoting behaviour change towards an MD diet. These findings provide important insights for the future development of high-quality apps to promote the MD at population level.

\section{Conflict of Interest}

There is no conflict of interest 